'sexting' as key term. We extracted data on; reasons for sexting, attitudes, and factors statistically associated with sexting.

Results Seven studies were included; most were cross-sectional, all were quantitative and conducted in the United States. Six studies assessed correlates of sexting in teenagers/young adults and found the following statistical associations; older adolescent, dating, sexually active, sexual risk behaviours, substance use, lower parental educational, peers sexting, and greater texting frequency. Girls were more likely to be senders, boys more likely to be receivers and to have asked someone to sext. Sexually active respondents were more likely to be both senders and receivers. Two studies explored attitudes about sexting finding those who sent pictures were more likely to consider sexting acceptable, over one third of non-sexters reported positive attitudes towards sexting, and most of those who sent pictures were bothered by having been asked to sext. Expecting serious legal consequences for getting caught sexting did not reduce reported sexting.

Conclusion Many young people don't perceive sexting negatively. Sexting may either be part of a cluster of risky sexual behaviours or in fact lead to sexual risk behaviour. Because of the cross-sectional nature of the studies, we were unable to determine causality. Additional research is needed to understand contexts in which sexting occurs, and motivations. Longitudinal designs are required to explore causality with sexual risk behaviour.

\section{P4.079 SEXUAL BEHAVIORS AND SAFETY STRATEGIES OF WOMEN WHO HAVE SEX WITH MEN AND WOMEN}

doi:10.1136/sextrans-2013-051184.0977

V R Schick, 'A Bell, 'C Neal, 'B Van Der Pol, 'B Dodge, 'L Bay-Cheng, ${ }^{3} \mathrm{~J} D$ Fortenberry. IIndiana University, Bloomington, IN, United States; ${ }^{2}$ University at Buffalo, Buffalo, NY, United States; Indiana University, Indianapolis, IN, United States

Background Women who have sex with women and men (WSWM) are at an increased risk for STI. Yet, knowledge regarding the behaviours associated with infection remains limited, with most studies focused on the experiences of women who have sex with only men or women. The diversity of sexual behaviours WSWM engage in may be limited by comparing WSWM to other groups. Instead, focusing on their experiences exclusively may provide a more comprehensive understanding of the sexual lives of WSWM

Methods Local (Indianapolis, IN, US) women who had engaged in recent genital contact with a male and female partner were invited to complete an on-line survey followed by an interview. Participants were asked to indicate the most recent time they had participated in a variety of behaviours with a male and/or female partner.

Results Eighty participants ranging in age from 18 to $51(M=26.74$, $\mathrm{SD}=7.97)$ completed the survey. The most commonly reported sexual behaviours were similar for male and female partners, including kissing, cuddling, external genital rubbing, vaginal fingering, cunnilingus/fellatio and penile-vaginal intercourse. While less commonly reported, a sizable minority of participants reported vaginal fisting, anal fingering and analingus. Toy use was reported by the majority of the participants with vibrator use reported as the most commonly used toy. Approximately $75 \%$ of participants indicated sexual behaviour with more than one person at one time. The percentage of participants who reported barrier use varied by behaviour and partner gender with the lowest percentage of participants reporting use during oral sex or genital-on-genital rubbing with a female partner (>90\% never) and the highest percentage reporting use during penile-vaginal intercourse ( $>25 \%$ always).

Conclusion Participants reported engaging in a variety of sexual behaviours that may facilitate STI transmission. Further knowledge about the types of behaviours WSWM engage in may help inform risk reduction strategies.

\section{P4.080 UNDERSTANDING THE INFLUENCE OF INDIVIDUAL AND PARTNER-SPECIFIC SEXUAL SEALTH ON SEXUAL RISK BEHAVIOUR AMONG ADOLESCENT WOMEN}

doi:10.1136/sextrans-2013-051184.0978

D J Hensel, J Fortenberry. Indiana University School of Medicine, Indianapolis, IN, United States

Background Sexual health identifies both individual and partnerspecific factors as important elements in public health approaches to STI prevention among adolescents, yet little empirical evidence links them to specific STI-related health outcomes.

Method Data were individual and partner-specific quarterly interviews from a cohort of young women in Indianapolis $(\mathrm{N}=387$, 14-17 yrs.). Using an existing sexual health definition (WHO, 2002) we created two standardised, multi-item sexual health scales: individual sexual health (sexual positivity, absence of genital pain, family communication, family connexion) and partner-specific sexual health (relationship satisfaction, sexual satisfaction, condom use self-efficacy, pregnancy prevention attitudes, sexual communication, partner's connexion to family) (both $\alpha \geq 0.85$ ). Outcomes were: used a condom at last sex (no/yes), ratio of condom-protected coital events, any sexual coercion (no/yes), current number sex partners $(2+/ 1)$, future number of sex partners (next 90-days: $2+/ 1$ ). Analyses were multilevel logistic and linear regression (HLM, 7.0; all $p<0.05$ ), overall and by current number of sexual partners.

Results Individual $(\mathrm{OR}=1.22)$ and partner-specific $(\mathrm{OR}=1.87)$ sexual health predicted condom use at last sex; partner-specific sexual health predicted no sexual coercion $(O R=0.69)$, a higher ratio of condom-protected coital events $(b=0.12)$, as well as having one sexual partner currently and for the anticipated future. Higher partner-specific sexual health predicted condom use at last sex in currently single $(\mathrm{OR}=1.70)$ and in currently multiple partner relationships $(\mathrm{OR}=2.22)$, a higher ratio of condom protected coital events in currently single $(b=0.15)$ and in currently multiple partner relationships $(b=3.66)$, and absence of sexual coercion $(\mathrm{OR}=0.19)$ in currently multiple-partner relationships.

Conclusion Individual and partner-specific sexual health are separately linked to key STI-related public health indicators. These data suggest that different elements may require emphasis to more fully support effective sexual health approaches to reducing STI in adolescents.

\section{P4.081 STD RISK PERCEPTION AMONG HIGHER RISK UNIVERSITY STUDENTS IN HALIFAX, CANADA}

doi:10.1136/sextrans-2013-051184.0979

\section{B Langille, A Steenbeek. Dalhousie University, Halifax, NS, Canada}

Background Perceived risk is central to health behaviour theory, though little is known about what creates perceived risk of contracting STDs. We examined self-rated risk of STD in higher sexual risk (HSR) university students in Halifax, Canada, to determine factors associated with recognition of such risk status.

Methods Using an online survey, we asked university students about their perception of their STD risk (greatly/quite a lot at risk versus not very much/no risk), their sexual behaviours, chlamydia knowledge (CK), friends' more liberal attitudes to sexual risk-taking (FLASRT), depression, and personal factors. HSR was defined as having had both $\geq 2$ partners for vaginal sex in the past year and no condom use at last intercourse. Variables initially associated with perceived HSR $(p<0.10)$ were entered into a logistic regression model controlling for gender to determine which remained associated with perception of being at HSR.

Results The survey response rate was $32 \%(\mathrm{~N}=4490)$, and 526 were at HSR. Of those with 2-5 partners in the previous year, only $14 \%$ rated themselves as at HSR, while $43 \%$ of those with $\geq 6$ 